\title{
La relación entre la asertividad y el emprendimiento
}

\section{The relationship between assertiveness and entrepreneurship}

DOI: $10.46932 / \mathrm{sfjdv3n1-087}$

Received in: Jan 30st, 2021

Accepted in: Feb 1th, 2022

\section{M.E. Alma Elizabeth Miranda Quiñones}

Profesora de Gestión empresarial en el Instituto Tecnológico de Tlalnepantla del Tecnológico Nacional de México, Tlanepantla, Estado de México.

E-mail: almaelizabeth2@gmail.com

\section{M.A.N. Ociel Núñez Sánchez}

Profesor de Plan de Negocios en el Instituto Tecnológico de Tlalnepantla del Tecnológico Nacional de México, Tlalnepantla, Estado de México

E-mail: ociel.ns@tlalnepantla.tecnm.mx

\section{Lic. Laura Quezada Mendoza}

Profesora de Gestión empresarial en el Instituto Tecnológico de Tlalnepantla del Tecnológico Nacional de México, Tlanepantla, Estado de México.

E-mail: laura.qm@tlalnepantla.tecnm.mx

\section{M.E. Guadalupe Fabiola Ramírez Solís}

Profesora de Gestión empresarial en el Instituto Tecnológico de Tlalnepantla del Tecnológico Nacional de México, Tlanepantla, Estado de México.

E-mail: guadalupe.rs@tlalnepantla.tecnm.mx

\section{M.C.E. Jasive Vilchis Guerrero}

Profesora de Gestión empresarial en el Instituto Tecnológico de Tlalnepantla del Tecnológico Nacional de México, Tlanepantla, Estado de México.

E-mail: jasive.vg@tlalnepantla.tecnm.mx

\section{M.A. Irma Violeta Loza Solache}

Profesora de Gestión empresarial en el Instituto Tecnológico de Tlalnepantla del Tecnológico Nacional de México, Tlanepantla, Estado de México.

E-mail: irma.1s@tlalnepantla.tecnm.mx

\section{M.E. Edna Nolasco Carlón}

Profesora de Gestión empresarial en el Instituto Tecnológico de Tlalnepantla del Tecnológico Nacional de México, Tlanepantla, Estado de México.

E-mail: edna.nc@tlalnepantla.tecnm.mx

\section{RESUMEN}

El documento pretende mostrar los resultados es determinar el nivel de asertividad relacionado con la competencia del emprendimiento en los alumnos de Ingeniería en Gestión Empresarial (IGE) del Instituto Tecnológico de Tlalnepantla, como herramienta para el desarrollo de las habilidades directivas tomando una muestra al azar de alumnos que se encuentran inscritos en el programa educativo de IGE y que se 
encuentran desarrollando proyectos de emprendimiento; para este fin se aplicó la Escala Multidimensional de Asertividad (EMA) que mide el grado de asertividad y el instrumento self-directed Search (SDS) que evalúa el nivel de emprendimiento dentro de la orientación vocacional, dando como resultado el factor de correlación entre la asertividad y el emprendimiento para coadyuvar en la productividad de las microempresas, presentando un diagnóstico prospectivo del desempeño como emprendedor.

Palabras clave: Asertividad, Escala Multidimensional de Asertividad (EMA), Instrumento EMA, SelfDirected Search (SDS) herramienta SDS, Emprendedor, Pequeñas y Medianas Empresas (PyMES).

\begin{abstract}
The paper aims to show the results is to determine the level of assertiveness related to entrepreneurship competence in students of Business Management Engineering (IGE) of the Instituto Tecnológico de Tlalnepantla, as a tool for the development of managerial skills by taking a random sample of students who are enrolled in the educational program of IGE and who are developing entrepreneurship projects; For this purpose, the Multidimensional Assertiveness Scale (EMA) that measures the degree of assertiveness and the self-directed Search (SDS) instrument that evaluates the level of entrepreneurship within the vocational orientation were applied, resulting in the correlation factor between assertiveness and entrepreneurship to contribute to the productivity of microenterprises, presenting a prospective diagnosis of the performance as an entrepreneur.

Keywords: Assertiveness, Assertiveness Multidimensional Scale (EMA), EMA Instrument, SelfDirected Search (SDS), SDS tool, Entrepreneur, Small and Medium Enterprises (SMEs).
\end{abstract}

\title{
1 INTRODUCCIÓN
}

Los avances tecnológicos han propiciado diversos cambios en el entorno de las organizaciones, la automatización de los procesos, digitalización de la información, la transfronterización a partir de las tecnologías de la información, los nuevos espacios de operación, etc., han acarreado alteraciones en la estructura decisional y en las relaciones horizontal y verticalmente, dando paso a nuevas necesidades de desempeño en los colaboradores, que aseguren la permanencia en el contexto empresarial, la reducción en los tiempos, la gran variedad de situaciones y la complejidad en los inconvenientes que se presentan en la actualidad, han dado cuenta del requerimiento de nuevas habilidades en los responsables de la toma de decisiones, quienes deben ser capaces de adecuarse y gestionar en ambientes complejos, estos cambios y nuevos retos han trascendido al nivel educativo superior, que deberá romper con paradigmas en la estructura de sus estrategias de formación, pasando del proceso enseñanza-aprendizaje a la simulación de escenarios reales, para lo cual se requiere como primer factor identificar la habilidad a desarrollar y el sistema de evaluación para validar resultados.

Entre las competencias que mayor impacto tienen al integrarse a la comunidad laboral, se encuentran las habilidades directivas, encargadas de diseñar y ejecutar acciones para transformar a la organización, lo que implica transmitir la idea y proceso que se desea seguir, alineando los objetivos para 
comunicarlos (Rivera, 2012) y expresar lo que necesita, siente y piensa, de forma consciente, clara y transparente, (Rodríguez-Sanz et al., 2016), para dirigir en la incertidumbre, situándose como un modelo de gestión emergente incidente para la competitividad empresarial, que genera expectativas y fortalece la identidad y el sentido de pertenencia (Moreno et al., 2014).

En situaciones de conflicto, con un alto requerimiento de respuesta, se vislumbra una relación con varios aspectos en la formación de estudiantes del nivel superior, de los cuales daremos referencia a dos de ellos: la capacidad de gestionar grupos de trabajo de manera eficiente y el generar nuevos planes de negocios, donde para la primera se requiere la habilidad de "articular las ideas con claridad para transmitir la visión" (Schmaltz, 2019, párr. 6) que de acuerdo a los estudios de Michel Page, la comunicación asertiva es una de las habilidades blandas que el directivo debe desarrollar; y con respecto a la segunda capacidad para identificar nuevos caminos, será necesario adaptarse y aprovechar las variaciones para generar nuevas ideas de negocio (Rengel, 2017), por lo que resulta interesante saber que tanto puede influir el expresar con claridad las ideas en la generación de proyectos y planes de negocios conocido también como emprendimiento.

La asertividad en palabras de Jardim y Pereira (2006, citado en Galinha y Sao-Joao 2021), es el comportamiento bilateral de dos personas, que enfatizan la importancia de la comunicación, de los deseos, pensamientos y sentimientos de ambas partes; lo que proporciona efectividad a la gestión empresarial, desde el punto de vista de Salter (2001 citado en Calua et al., 2021) la asertividad permite a las personas, actuar con seguridad y resolver problemas de manera constructiva. En otro sentido el desarrollo de una idea y ponerla en práctica dentro de una organización para hacerla crecer describen las competencias emprendedoras, (Deza-Loyaga et al., 2021), por su parte, Casimiro et al. (2019), define al emprendimiento como una estrategia de generación de innovación y de competitividad en la industria, adicionalmente, Sumba y Santiesteban, (2018) mencionan que las microempresas se originan correspondiendo a la necesidad de creación de empleos, capaces de transformar habilidades y aptitudes, logrando emprendimientos que generan la posibilidad de mejorar las condiciones de vida de las familias del personal que labora en la organización, y en comunión con la asertividad logar una relación adecuada con sus colaboradores, quienes aportan sus competencias para lograr el éxito organizacional (Rengel, 2017).

Ante esto se ha considerado de gran relevancia identificar como la asertividad impacta de manera directa en el nivel de emprendimiento, de donde se despende el objetivo de esta investigación, que se centra en determinar laa correlación existente entre asertividad relacionado con la competencia del emprendimiento en los alumnos de Ingeniería en Gestión Empresarial (IGE) del Instituto Tecnológico de Tlalnepantla, para aplicarse como herramienta para el desarrollo de las habilidades directivas y como parte de las estrategias para generar autoempleo en los futuros profesionales, la investigación es inicialmente 
de tipo cualitativo para posteriormente ser trasladado al corte experimental-cuantitativo, empleando instrumentos validados, como es la Escala Multidimensional de Asertividad (EMA) para trabajar la variable independiente, que pretende identificar el grado de comunicación interpersonal y el Self-Directed Search (SDS) orientación vocacional; precisando la relación con la capacidad de emprendimiento que se observa como variable dependiente.

\subsection{ESTUDIOS PREVIOS}

La evolución en el grado de complejidad, incremento de volúmenes y calidad de la productividad y servicio, ha obligado a las organizaciones a replantear sus modelos de gestión, teniendo como herramienta a la comunicación para la generación de valor en activos intangibles (Marín-Dueñas, 2015), por su parte Muñoz (2012), considera que la comunicación es un factor relacional que incide en la productividad de las pymes, y que para operacionalizar los objetivos se requiere de habilidades efectivas de comunicación, persuasión, relaciones humanas y negociación Garbanzo-Vargas, (2016), de acuerdo a Moreno et al., (2014) al existir un reforzamiento en la comunicación entre el personal operativo y el gerencial se fortalece el sentido de pertenencia, viéndose reflejado en la productividad, sin embargo aun cuando la comunicación se ha convertido en una herramienta de generación de valor para las empresas según (Marín Dueñas, 2015); Dupleix y Rébori, (2017), detectan que uno de los factores que afectan la permanencia de las pymes en el mercado, es la reorientación de las estrategias, presentando la imposibilidad por parte del cuerpo directivo para llevar a cabo su alineación y ejecución, reafirmando la postura de Marín-Dueñas (2015) respecto a que las pymes no suelen utilizar esta perspectiva.

Pese a que una gran cantidad de países se encuentran centrados en dar apoyo a las pymes por considerar que es una estrategia para disminuir la crisis económica, estas no surten efectos, debido a que el enfoque se encuentra centrado en el financiamiento, producción, innovación y desarrollo y en menor intensidad hacia los modelos de gestión; entre las causas que originan el fenecimiento de las pymes, dentro de los primeros cinco años; se encuentra el empirismo con que inician sus gestiones los empresarios, en términos de habilidades gerenciales (Instituto Nacional del Emprendedor (INADEM), 2016) por su parte Andriani et al., (2004), agrupan las deficiencias de las pymes en 1). Los sistemas de gestión, 2). Los procesos, 3). El lado humano y 4). El sistema de información y diagnóstico (Castañeda, 2013).

Ante lo anterior y considerando el ambiente de educación superior, se identifica como un área de oportunidad establecer un programa que detone el interés en los jóvenes para desarrollarse en el ambiente del emprendimiento, a partir de la aplicación de las herramientas EMA y SDS en los estudiantes de gestión empresarial, tendientes a lograr las siguientes metas: 1. Provocar un acercamiento de los alumnos hacia las herramientas, 2. Extender una campaña sobre el emprendimiento y su factibilidad, 3. Mostrar a los 
educandos en lo individual el grado de desarrollo de las habilidades de asertividad y emprendimiento.

\section{DESCRIPCIÓN DEL MÉTODO}

La investigación se define como cualitativa, ya que de acuerdo con Bernal (2020) se centra en entender la situación social, partiendo de la conceptualización de la realidad, apoyado en la información que se obtiene a través de la aplicación de un instrumento, realizada generalmente en pequeñas muestras y visto como un estudio de caso, (Vara-Horna, 2012) seleccionándose éste diseño por ser aplicado en una institución educativa e incluso en el área de administración según lo menciona (Hernández-Sampieri, 2014), de este modo y como lo indica el enfoque cualitativo en el presente proyecto, se tiene la intención de describir las características de la muestra de jóvenes universitarios como emprendedores, haciendo uso del método cuantitativo para contrastar la hipótesis planteada a través de herramientas estadísticas e instrumentos estructurados y validados aplicados en una muestra representativa de la comunidad (Paniagua-Machicao y Condori-Ojeda, 2018).

Se seleccionó un grupo de 35 alumnos al azar, los criterios utilizados para su integración como parte de la investigación, se estructuraron bajo tres premisas a). Estar inscritos en el programa educativo de ingeniería gestión empresarial (IGE), b). Haber entregado proyecto de emprendimiento para su validación y c). El interés hacia la metodología de aplicación de los instrumentos y el proceso de análisis e interpretación de los resultados, una vez concluida la primer etapa se procedió a la aplicación de la Escala Multidimensional de Asertividad (EMA), y el Self-Directed Search (SDS) orientación vocacional, ambos cuestionarios cuentan con el proceso de validez y confiabilidad, el caso de EMA permite evaluar tres dimensiones: no asertividad, asertividad indirecta y asertividad (Miranda, Vargas y Becerril, 2016), mientras que el SDS presenta resultados sobre la orientación vocacional, detectando la relación entre las destrezas y el área de formación decidida por el estudiante. La tercera y cuarta etapa consistieron en la recolección de los datos obtenidos de la población evaluada, de los instrumentos EMA y SDS, para el análisis e interpretación cuantitativa y obtener así el nivel de asertividad relacionado con la competencia del emprendimiento de alumnos inscritos en el programa educativo de IGE del Instituto del Tecnológico de Tlalnepantla, como herramienta para el desarrollo de las habilidades directivas.

\subsection{DESCRIPCIÓN DE LAS METODOLOGÍAS}

La herramienta EMA fue diseñada y validada por Flores y Díaz-Loving en 2004, con la intensión de identificar de entre las tres dimensiones cual cuenta con el grado de mayor influencia, está estructurada por 45 afirmaciones y cada una con cinco opciones de respuesta tipo Likert, los autores describen la no asertividad, asertividad indirecta y asertividad para describir la capacidad para dar a conocer los 
sentimientos, pensamientos, opiniones, dar y recibir alabanzas, manejo de crítica e interacción con otras personas, así la no asertividad se considera como la ausencia de habilidad para manejar los factores, mientras que la asertividad indirecta, muestra a las personas que no pueden manejar los descriptores encarando a los interlocutores y la asertividad es precisamente el dominio para poner en práctica todos los elementos en conjunto (Miranda et al., 2016).

El instrumento (SDS) orientación vocacional diseñado por Holand en 1971 su más reciente actualización es en 1997; describe la conducta del individuo en seis áreas de la personalidad, por medio de patrones distintivos relacionados con el ambiente, el cual se ajusta a su comportamiento así las dimensiones son: realista (R), prefiere tareas relacionadas con la manipulación de objetos, herramientas, máquinas y animales; el tipo investigador (I) prefiere actividades que conducen a la observación, a lo simbólico, a lo sistemático; artístico (A), implican una manipulación de materiales físicos, verbales y humanos para crear formas y productos; social (S), describen comportamientos de interacción con otras personas con la finalidad de informar, curar, educar e instruir; emprendedor (E), el dominio es lograr objetivos organizativos y ganancias económicas; y convencional (C), sus metas de vida gustan por producir mucho trabajo y volverse expertos en las finanzas (Martínez, 2007).

De la misma manera y como método de análisis de relación de variables se utilizará la correlación de Coeficiente de Pearson, la cual será útil para medir el grado o asociación existente entre las dos variables (Restrepo 2007), presentadas para este caso asertividad y emprendimiento cuyo nivel de significancia estará calculado a través de la herramienta minitab como software de análisis de datos.

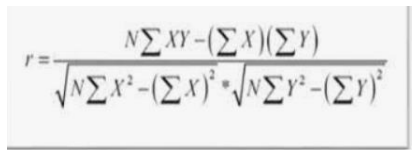

Donde:

$\mathrm{N}=$ Tamaño de la muestra

r=correlación de Pearson ó Pvalor, nivel de significancia

Nivel de significancia:

Mayor a 0.05 significa que no existe relación entre las variables

Igual o menor a 0.05 significa que hay una relación significativa entre variables

Igual o menor a 0.01 significa que hay una relación MUY significativa entre variables

\subsection{INTERPRETACIÓN DE LOS RESULTADOS}

El procedimiento del análisis de resultados se desarrolla a partir de la aplicación de los instrumentos, utilizando métodos estadísticos básicos en la determinación media para conocer el promedio dentro de la muestra con respecto a los aspectos de asertividad, asertividad indirecta y no asertividad. 


\section{RESULTADOS}

\subsection{ESCALA MULTIDIMENSIONAL DE ASERTIVIDAD (EMA)}

El cuestionario se aplicó a 35 alumnos que cubrían los tres elementos característicos: a). Estar inscritos en el programa educativo de ingeniería en gestión empresarial, b). Haber entregado proyecto de emprendimiento para su validación y c). El interés hacia la metodología de aplicación de los instrumentos y el proceso de análisis e interpretación de los resultados; el reporte de la aplicación del cuestionario EMA, presentado en el Cuadro1 proporcionó las valoraciones estadísticas correspondientes a cada dimensión (asertividad, asertividad indirecta y no asertivo), siendo el previo para llevar a cabo el proceso de interpretación de la información arrojada por el constructo (se recomienda revisar el proceso completo de aplicación de EMA en el antecedente realizado por Miranda et al., 2016), de acuerdo a estos datos se realizó la transferencia para su interpretación (cuadro 1) y en la Gráfica 1, obteniéndose que 16 alumnos de 35 el $45.71 \%$ cuentan con la habilidad de tener una comunicación efectiva y adecuada para transferir los pensamientos e ideas de manera clara, esta es una de las habilidades que se debe de fortalecer dentro de emprendedor, el $28.57 \%$ tienen la característica de comunicarse de forma asertiva.

Cuadro 1. Promedios EMA

\begin{tabular}{crrr}
\hline Subescala & Promedio & Mediana & Moda \\
\hline Asertividad & 57.1714 & 57 & 57 \\
Asertividad & 48.3714 & 50 & 54 \\
Indirecta & & & \\
$\quad$ No & 47.1714 & 49 & 36 \\
Asertividad & & & \\
\hline
\end{tabular}

Gráfica 1. Porcentajes EMA

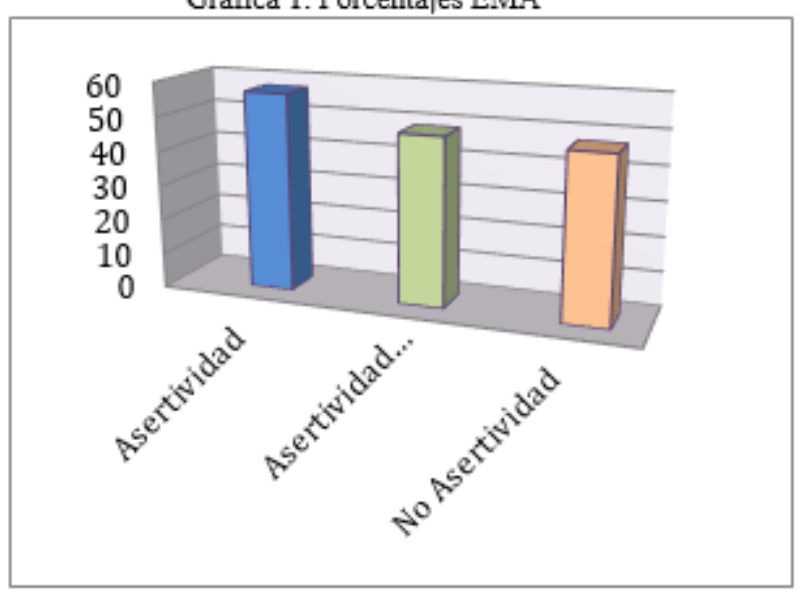

\subsection{SELF-DIRECTED SEARCH (SDS) ORIENTACIÓN VOCACIONAL}

De acuerdo a los cuadernos de autoevaluación que fueron aplicados al alumnado, se presenta la Gráfica 2 en la que se visualiza el porcentaje de los estudiantes con la orientación a ser emprendedores con un $66 \%$ de alumnos que se encuentran con esta visión, seguidos con 23\% hacia la orientación convencional, $5.71 \%$ con ocupaciones realistas, es decir, técnicas, manuales de servicio, $2.85 \%$ con personalidad artística para actividades literarias y música, y el mismo porcentaje con habilidades sociales como son actividades educativas y servicio social y $0 \%$ con características de investigadores. 
Grafica 2 Porcentajes de SDS

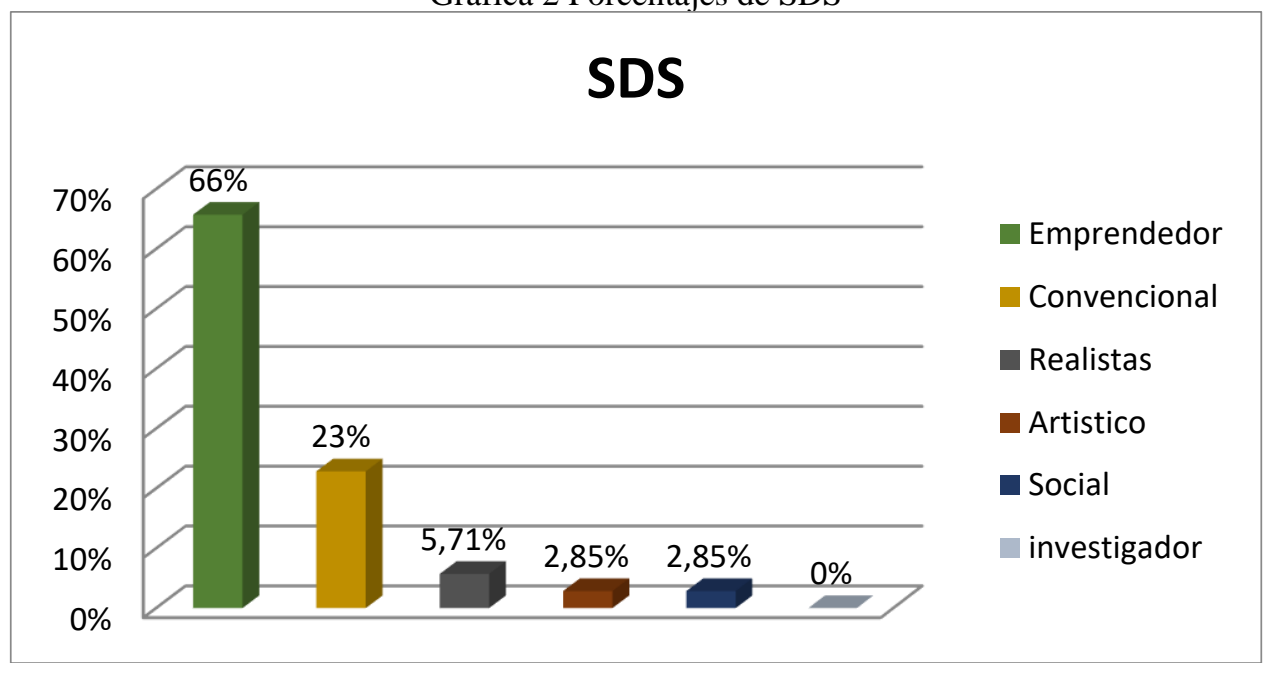

Análisis e interpretación de correlación de Pearson, para tal efecto a continuación se presenta el siguiente análisis de correlación de Pearson que nos mostrara el nivel de alcance correlacional entre las variables para este caso emprendimiento variable dependiente y asertividad como variable independiente y su nivel de significancia, es decir el grado de impacto, tal como se describe al inicio de este artículo.

\begin{tabular}{|c|c|c|}
\hline \multirow{2}{*}{ Correlación } & . Correlación de datos & \\
\hline & $\begin{array}{l}\text { Variable Relacional } 1 \\
\text { Independiente } \\
\text { Asertividad }\end{array}$ & $\begin{array}{l}\text { Variable Relacional } 2 \\
\text { Dependiente } \\
\text { Emprendimiento }\end{array}$ \\
\hline $\begin{array}{l}\text { Variable Relacional } 1 \\
\text { Independiente } \\
\text { Asertividad: }\end{array}$ & & \\
\hline $\begin{array}{l}\text { Correlación de Pearson } \\
\text { Nivel de Significancia }\end{array}$ & 1 & 0.815 \\
\hline $\mathrm{N}$ & 35 & 35 \\
\hline $\begin{array}{l}\text { Variable Relacional } 2 \\
\text { Dependiente } \\
\text { Emprendimiento: }\end{array}$ & & \\
\hline $\begin{array}{l}\text { Correlación de Pearson } \\
\text { Nivel de Significancia }\end{array}$ & 0.661 & 1 \\
\hline $\mathrm{N}$ & 35 & 35 \\
\hline
\end{tabular}

Dado que el valor del Pvalor de correlación y nivel de significancia es mayor a 0.05 , entonces hay una relación directa entre el nivel de asertividad al impactar al emprendimiento de manera directa, por lo tanto, la hipótesis inicial se cumple de manera verdadera. 


\section{RESUMEN DE RESULTADOS}

De esta investigación y conforme a la metodología descrita se obtuvieron los resultados de ambos instrumentos facilitando su integración e interpretación cuantitativa del nivel de asertividad que se busca en el alumnado del ITTLA, en relación a la definición del emprendimiento y enfoque empresarial, resaltando un $66 \%$ de los alumnos con una visión emprendedora, la cual se complementa con competencias directivas y facilitar la productividad versus inestabilidad, así como una comunicación asertiva que favorece el sentido de pertenencia, afrontando la incertidumbre mediante modelos de gestión emergente. El 40\% del alumnado carece de la habilidad de comunicación asertiva, quedando el 60\% con la visión de emprender un negocio.

\section{CONCLUSIONES}

Se aplicó el cuestionario de la Escala Multidimensional de Asertividad (EMA), y el Serlf Directed Search (SDS) orientación vocacional, a 35 alumnos que cubrían tres factores: a). Estar inscritos en el programa educativo de gestión empresarial, b). Haber entregado proyecto de emprendimiento para su validación y c). El interés en participar de la aplicación de los instrumentos. El proceso de análisis e interpretación de los resultados arrojo que la media de la muestra cuentan con un desarrollo de las habilidades emprendedoras, es decir resalta su visión o enfoque empresarial, con referente al desarrollo de las habilidades directivas para la toma de decisiones, solución de conflictos, gestión y formulación de proyectos e implementación de modelos de gestión emergente para incrementar la productividad y afrontar la incertidumbre socioeconómica empresarial han sido desarrollado tomando como base los resultados obtenidos en el cuestionario SDS.

La correlación del nivel de asertividad y el emprendimiento, son dos de las habilidades que requieren ser detectadas oportunamente en todo individuo en formación educativa o empresarial, para su orientación y ocupación en el mercado laboral, haciendo imprescindible la habilidad efectiva de comunicación en toda socialización, que contrarresta las deficiencias que originan el fenecimiento de las pymes. Dentro de los primeros cinco años se encuentra el empirismo con que inician gestiones los empresarios en términos de sus habilidades gerenciales.

Parte de los factores que intervienen en el liderazgo se encuentra la comunicación asertiva, la cual permite generar un grado de confianza entre los colaboradores y seguridad al realizar las funciones que le corresponden, resultado de la intercomunicación que ejerce el líder, así como la capacidad de visionar proyectos de mejora en la organización y/o desarrollo de innovaciones para generar nuevas empresas. 
La prospección resultante en esta investigación permitirá el mejorar las estrategias de fortalecimiento de la cultura del emprendimiento en la formación de los profesionistas del Instituto Tecnológico de Tlalnepantla, partiendo del diagnóstico e intervención mediante un programa de capacitación en las áreas de oportunidad detectadas, mediante las herramientas Ema y SDS, atendiendo el desarrollo de las habilidades de asertividad y emprendimiento requeridas por esta comunidad.

\section{RECOMENDACIONES}

Los resultados demuestran la necesidad de implementar estrategias de capacitación, difusión y fortalecimiento de la cultura del emprendimiento, como catalizador para el desarrollo de la comunicación asertiva a los estudiantes que no han desplegado esta habilidad y fortalecer aquellos que ya cuentan con esta destreza, ya que el perfil que se debe de tener dentro de la Institución en IGE requiere de ellas, para diseñar y emprender nuevos negocios y proyectos empresariales. Una comunicación asertiva con enfoque empresarial facilita relaciones comerciales que hoy en día son un auge en la era de la tecnología y globalización. 


\section{REFERENCIAS}

Alexandre Galinha, S., \& São-João, R. (julio de 2021). Periodo Covid-19 y competencias en la educación superior -estudio sobre cooperación y asertividad. South Florida Journal of Development, 2(3), 41794187. doi:10.46932/sfjdv2n3-031

Bernal, C. (2020). Metodología de la investigación. Tercera edición. Pearson Educación.

Cadena-Badilla, J., \& Hernández León, R. (junio de 2021). Perfil de emprendimiento de los estudiantes de la Universidad de Sonora, Unidad Norte de México. South Florida Journal of Development, 2(2), 31003117. doi:10.46932/sfjdv2n2-149

Calua, M., Delgado, Y. \& López, O. (2021). Comunicación asertiva en el contexto educativo: revisión sistemática. Revista Boletín Redipe, 10(4), 315-334. Disponible en: https://dialnet.unirioja.es/servlet/articulo?codigo $=7917878$

Castañeda, L. (2014). Los sistemas de información contable una herramienta para la gestión integral de las pymes. Revista Gestión Joven, 11, 31-40. Disponible en: http://www.elcriterio.com/revista/contenidos_11/luz_idalia.pdf

Deza-Loyaga, W. F., Aparicio-Ballena, J. A., Pérez-Arboleda, P. A., y Hidalgo-Lama, J. A. (2021). Competencias de emprendimiento en estudiantes universitarios en Perú: metodología para su desarrollo. Revista Venezolana de Gerencia, 26(96), 1172-1188. https://doi. org/10.52080/rvgluz.26.96.11

Casimiro Urcos, W. H., Casimiro Urcos, C. N., \& Casimiro Urcos, J. F. (2019). Competencias de emprendimiento empresarial en estudiantes universitarios. Universidad y Sociedad, 11(5), 61-69. Disponible en: http://rus.ucf.edu.cu/index.php/

Dupleix, M. y A. Rébori. (2017). La estrategia y la trasformación de firmas en declive: revisión de la literatura en los últimos 15 años. Revista Estudios Gerenciales, 33 (143), 141-152. Doi: http://dx.doi.org/10.1016/j.estger.2017.02.005

Garbanzo-Vargas, G. (2016). Desarrollo organizacional y los procesos de cambio en las instituciones educativas un reto de la gestión de la educación, Revista Educación. 40 (1), 67-87. Disponible en: http://www.redalyc.org/articulo.oa?id=44043204005

Hernández-Sampieri, R., Fernández-Collado, C., Baptista-Lucio, P. (2014). Metodología de la investigación. Sexta edición. McGraw Hill Educación.

Marín-Dueñas, P. (2015). Comunicación empresarial en las pequeñas y medianas empresas (pymes) percepción, conocimiento y actitud, Revista Mediterránea de Comunicación. 6 (2), 63-82. Doi: 10.14198/MEDCOM2015.6.2.03

Martínez, J.M. (2007). El asesoramiento vocacional y profesional a través de Self-Directed Search, Revista Electrónica de Investigación Psicoeducativa. 5(1), 233-258. Disponible en http://www.investigacion-psicopedagogica.org/revista/articulos/11/espannol/Art_11_166.pdf

Miranda, A., Vargas, M. y Becerril, E. (2016). Análisis de la aplicación de la herramienta EMA, en estudiantes de ingeniería en gestión empresarial, Memorias de congreso en el Libro electrónico: Gestión Social Organizaciones Humanas para una Sociedad Global Incluyente, 2016, Disponible en: http://acacia.org.mx/wp-content/uploads/2016/11/EBook_XX_Congreso_ACACIA.pdf 
Moreno, A., Arbeláez, S. y Calderón, L. (2014). Implementación de herramientas de comunicación interna como generadoras de cambios en las pymes, Revista Razón y Palabra, 18(88), 1-27. Disponible en: http://www.redalyc.org/articulo.oa?id=199532731009

Muñoz, M. (2012). Comunicación y productividad en pequeñas y medianas empresas de un cluster textil en Colombia, Revista Contaduría y Administración 57(2) 223-244. Disponible: http://www.scielo.org.mx/scielo.php?script=sci_arttext\&pid=S0186-10422012000200011

Paniagua-Machicao, F. y Condori-Ojeda, P. (2018). Investigación científica en educación. Juliaca: Porfirio Condori Ojeda (autor-editor). Disponible en. https://www.aacademica.org/cporfirio/

Rengel, A. (2017, agosto 16). Emprendimiento y liderazgo. [Mensaje de Blog]. Insights, Knowledge Driven Content. https://www.ie.edu/insights/es/articulos/emprendimiento-yliderazgo/\#: :text=Los\%20verdaderos\%201\%C3\%ADderes\%20emprendedores\%20son,el\%20comprom iso $\% 20$ con $\% 20$ sus $\% 20$ ideas.

Restrepo, L. y González, J. (2007). De Pearson a Spearman. Revista Colombiana de Ciencias Pecuarias, 20(2), 183-192. Disponible en: https://www.redalyc.org/articulo.oa?id=295023034010

Rivera, A. (2012). Nestlé Comunicación que trasciende, Entrevista realizada por J. Tolentino, marzo 2012. Disponible en: artículos/artículos\%20diversos/Tolentino\%202012\%20comunicación\%20 asertiva.pdf

Rodríguez-Sanz, J., Álvarez-Ude, F. y Estrella-Gimeno, V. (2016). Lo esencial en la asertividad, Revista Formación Médica Continuada en atención primaria. 23(6), 352-357. Doi. https://doi.org/10.1016/j.fmc.2015.11.009

Schmaltz, C. (2019, abril 3). Cómo ser un buen líder para tu empresa [Mensaje de Blog]. Red Forbes. https://www.forbes.com.mx/como-ser-un-buen-lider-para-tu-empresa/

Sumba-Bustamente, R. Y. \& Santiesteban-Villacreses, K.I. (2018). Las microempresas y la necesidad de fortalecimiento, reflexiones de la zona sur de Manabí, Ecuador. Universidad y Sociedad, 10 (5), 323-326. Disponible en: http://scielo.sld.cu/pdf/rus/v10n5/2218-3620-rus-10-05-323.pdf 